Books, videos, cd-roms, dvds and any other relavent items submitted for a review in the $B D J$ should be addressed to:

Mike Grace, Editor, British Dental Journal, 64 Wimpole Street WIG 8YS
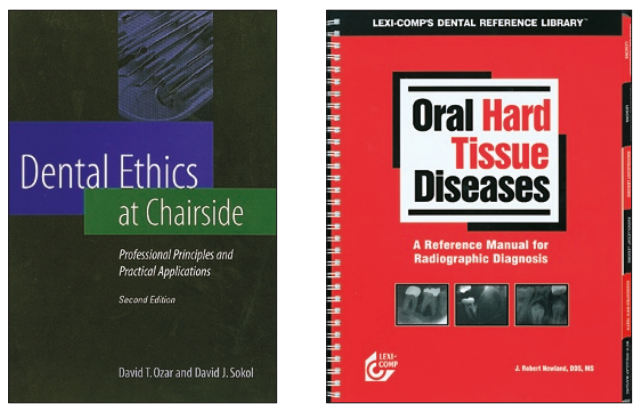

\section{Dental ethics at chairside: professional principles and practical applications}

\author{
D. T. Ozar, D. J. Sokol \\ Washington, DC: Georgetown University Press, 2002. \\ 2nd ed \\ price US\$42.50, pp272 \\ ISBN 0878403760
}

Ethics in healthcare has become a vital issue for related professions when so many changes appear to be occurring simultaneously. Examples are easily drawn together, such as the changing nature of oral disease, increasing patient expectations, third-party payers or managed care, whistle blowing by colleagues, consent for treatment to name a few. Hence, the arrival of the second edition of an already well-known text on dental ethics is especially welcome.

The book consists of 15 chapters and is divided into two parts. The first part of the book covers the dental profession and professional ethics. Nine categories of professional obligation are outlined. The authors present four models of the dentist-patient relationship. The similarity with models from a behavioural science perspective confirmed for me the grounding of the analysis presented by Ozar and Sokol. The conclusion of the authors within this part of the book is to recommend the interactive model as closest to matching the translation of a dentist's central values into the workplace and assist in reflecting joint decisions with their patients about how to conduct ethical practice.

The second part of the book, consisting of 10 chapters, presents the bulk of the key ethical problems and questions that are likely to face the career dentist. These include: working with compromised and anxious patients, collaborating with other professionals, issues of confidentiality and social justice. These areas are presented skilfully through the use of cases and enable the authors to weave their considerable expertise and experience to illuminate the challenges that await the dental professional. Considerable thought has been expended to develop these scenarios and help to develop and illustrate their model of professional-ethical decision making.

I was surprised at the lack of references, which does help to keep the reader engaged with the arguments and prevents distraction. However a detailed and annotated bibliography appears at the end of the text. The second edition consists of two completely new chapters on record keeping, electronic storage and the advent of managed care programmes. An interesting final chapter on the broader aspects of ethics in the dental profession and the community, invites the profession at all levels to engage the public to ensure that the services offered by the profession adequately reflects the demands of a modern society. The book has been written in North America and hence does not make reference to professional organisations in Europe, however the measured and considered approach adopted by these authors should guarantee that this text is required reading for any serious dental professional wishing to maintain excellent relationships with the communities they wish to serve. G. Humphris

\section{Oral hard tissue diseases}

\section{J. R. Newland \\ Ohio: Lexi-comp Inc, 2003 \\ price US\$44.95, pp 107 \\ ISBN 1591950287}

This book is aimed at 'the busy dental practitioner' and forms part of a series of soft cover, ring-bound manuals on a range of clinically important subjects. The author is a teacher at the University of Texas Dental School who also runs a private practice. The purpose of the book is to present radiographic features of intrabony lesions discovered on dental radiographs. If you have ever found something on a radiograph and wondered 'what's that?' then this may be the book for you.

There are 11 sections separated by tagged dividers for easy searching. The sections are based upon basic radiological features eg 'periapical radiolucent lesions' and 'inter-radicular radiopaque lesions'. In each case, material is described in a systematic way, illustrated by mainly good quality images and divided into common and less common lesions. The approach is logical, with each lesion being described under sub-headings of 'synonyms', 'aetiology', 'typical radiographic features', 'useful clinical information', 'differential diagnosis', 'diagnostic steps', 'treatment recommendations', 'follow up' and 'clinical significance'. There is an excellent list of references at the end of the book.

There were a few things that niggled me. The most important is that because some lesions present in various radiological guises, for example a cementoma, they can appear in more than one section in the book. It would have been easy to crossrefer between sections to demonstrate this when it happens. A most important task in interpretation of radiographs is deciding when normal becomes abnormal; thus it would have been nice to have a size guide as to when a nasopalatine foramen might be considered cystic or a tooth follicle a dentigerous cyst. I do not like to diagnose an 'abscess' on a radiograph - this is a clinical diagnosis. I would certainly take issue with a need to biopsy a marrow space to confirm diagnosis. One of the images on page 37 is certainly wrong.

I suspect that this book is a labour of love based upon years of teaching undergraduates and is founded upon a great collection of radiographs. It would be valuable to all sorts of people, not just for everyday use by dentists, but also for undergraduates, for those preparing for MFDS exams and just as general CPD material. Even bearing in mind the odd contestable statement and limitations described above, I think this book is very valuable and should be in any dental school library.

\section{K. Horner}

